\title{
A New Way to look at Observations with EGSO
}

\author{
J. Aboudarham ${ }^{1}$, I. Scholl ${ }^{2,1}$, N. Fuller ${ }^{1}$, A. Csillaghy ${ }^{3}$, R.D. Bentley ${ }^{4}$, \\ E. Antonucci ${ }^{5}$, L. Ciminiera ${ }^{6}$, A. Finkelstein ${ }^{7}$, S. Ipson ${ }^{8}$, \\ M. Messerotti ${ }^{9}$, D. Pike ${ }^{10}$, J.C. Vial ${ }^{11}$ and V. Zharkova ${ }^{12}$ \\ ${ }^{1}$ LESIA, Observatoire de Paris, 5 place Janssen, F-92190 Meudon, France \\ email: Jean.Aboudarham@obspm.fr \\ ${ }^{2}$ I.S.U., Strasbourg, France, ${ }^{3}$ U.A.S, Brugg, Swizterland, ${ }^{4}$ M.S.S.L., U.C.L., Great Britain, \\ ${ }^{5}$ Osservatorio di Torino, Italy, ${ }^{6}$ Politecnico di Torino, Italy, ${ }^{7}$ C.S., U.C.L., Great Britain, \\ ${ }^{8}$ Cybernetics, Univ. Bradford, Great Britain, ${ }^{9}$ Osservatorio di Trieste, Italy, ${ }^{10}$ R.A.L., Great \\ Britain, ${ }^{11}$ I.A.S, Orsay, France, ${ }^{12}$ Cybernetics, Univ. Bradford, Great Britain
}

\begin{abstract}
The European Grid of Solar Observations (EGSO) is a Solar virtual observatory (see Hill et al., 2002). It has been funded through the 5th Framework Program of the European Community. A dozen of laboratories, mixing Solar Physics and Information Technology, in Great Britain, France, Italy and Swiss have been involved in this project during 3 years. A grid accessing several dozens of databases and archives scattered all around the world has been developped as well as a Solar Event Catalogue and a Solar Feature Catalogue. The original aspect of this work consists in the possibility not only to search through the characteristics of observations, but also search for available data corresponding to specific kinds of events. So it is now very important to be able to follow the Sun 24 hours a day in order to enrich the events database for future queries. More informations on EGSO, catalogues and user interface can be accessd through the web site: http://www.egso.org/
\end{abstract}

Keywords. catalogs, methods: data analysis, Sun: general

\section{Introduction}

At the moment, Solar observations are scattered all around the world. Some are available through databases or FTP, some archives can be accessed through Internet, some don't.. Even if many instruments now use FITS format for the observations, the definition of keywords may vary strongly from one instrument to the other. Some beginning of normalization, but still in progress, occured with space missions since YOHKOH, especially with SOHO, as well as in France for ground-based observations, with BASS 2000 (http://bass2000.obspm.fr/home.php). All this makes it difficult to look for simultaneous observations coming from different sources. So there is a need of simplification of the access to data. This simplification is what we call a virtual observatory. It's some kind of layer that allows the user to see the whole accessed archives as a unified consistent catalogue where, with a simple query, he can retrieve the interesting data from various instruments.

\section{What is EGSO?}

EGSO is the acronym of European Grid of Solar Observations. It's a virtual solar observatory. 
It's a project funded by the European Community from March 2002 to June 2005. It's a computer grid, in the Computer Science meaning of this term. And it proposes important added values to archives available, which are:

New catalogues and Data Model (taking into account specificities of Solar physics)

Meta-data exchange format compatibles with other VOs (xml format organised in VOTables)

Sofisticated query and visualization services

No constraints for archives to be accessed by EGSO.

The coordinator of the project was R.D. Bentley, from M.S.S.L. Twelve laboratories from 5 countries were involved in EGSO:

In Great Britain:

University College London: UCL-MSSL and UCL-CS

Rutherford Appleton Laboratory

University of Bradford

In France:

Observatoire de Paris-Meudon

Institut d'Astrophysique Spatiale (Orsay)

International Space University (Strasbourg)

In Italy:

Istituto Nazionale di Astrofisica

Politecnico di Torino

INAf, including Observatories of Turin, Trieste, Florence and Naples

In Switzerland : University of Applied Sciences (Aargau)

In USA:

Solar Data Analysis Centre at NASA-GSFC (Greenbelt, MD)

National Solar Observatory (Tucson, AZ)

Eighteen full time equivalents have been working during three years to develop this project.

Close collaborations were maintained with COSEC (Collaborative Sun Earth ConnectorSolarSoft Services); VSO (Virtual Solar Observatory, including SDAC and NSO); and VSPO (Virtual Space Physics Observatory).

\section{The Database Grid}

Three roles make up the grid: Consumer, Broker and Provider roles.

The Consumer role assumes the management of the relationship with the user which can be either connected through graphic user interface (GUI) or directly, using IDL software, where a package is available in the SolarSoft package, 'vobs' branch, 'egso' sub-branch. The Provider role manages the connection with all the various sources of data. Those sources can be the archives that EGSO can access, or internal informations such as those concerning data model, description of data sources, and so on. Last, but not least, the Broker role is the intelligent part of the grid: it connects the consumer role to the provider role in an intelligent way, ensuring that each role can understand the other.

Figure 1 shows the links between the three roles. 


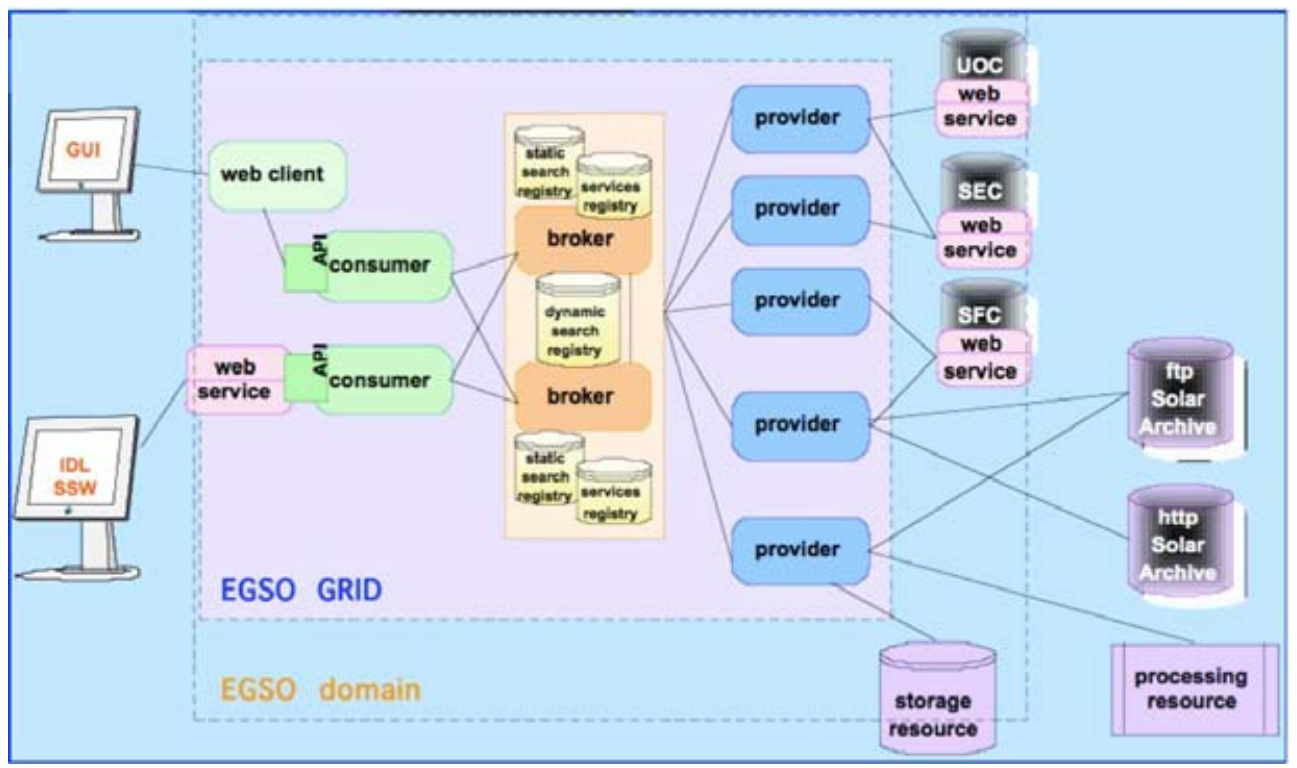

Figure 1. Grid architecture (see text for details).

\begin{tabular}{|c|c|c|c|c|c|c|}
\hline CATALOGUE & NAME & TYPE & FROM & TO & STATUS & RECORDS \\
\hline GEV GOES event list & goes_xray_flare & event & 1975-Sep-01 & 2005-Feb-24 & active & 60050 \\
\hline Solar $\mathrm{H}$-alpha Flare events & halpha_flares_event & event & 1980-Jan-01 & 2004-Deo-31 & active & 188495 \\
\hline NOAA SGAS Energetic Events & sgas_event & event & 1996-Jan-04 & 2005-Mar-20 & active & 7373 \\
\hline Yohkoh HXT Flare List & yohkoh_flare_list & event & 1991-Oct-01 & 2001-Dec-14 & closed & 3112 \\
\hline HESSI Flare List & hessi_flare & event & 2002-Feb-12 & 2005-May-14 & active & 15384 \\
\hline Kanzelhoehe Flare List & kso_flare & event & 1984-Jan-02 & 2005-May-13 & active & 8233 \\
\hline EIT Waves & eit_list & list & 1997-Mar-25 & 1998 Jun-16 & closed & 460 \\
\hline Yohkoh SXT TAACE flare list & yohkoh_sxt_trace_list & list & 1900-Jan-22 & 1999-Dec-27 & closed & 392 \\
\hline NOAA Proton Events & noaa proton_event & event & 1976-Apr-30 & 2005-Jan-16 & active & 216 \\
\hline LASCO CME Catalogue & lasco_cme_cat & event & 1996-Van-11 & 2004-Dec-31 & active & 9149 \\
\hline LASCO Preliminary CME List & lasco_cme_list & event & 1900-May-26 & 2005-Apr-27 & active & 8178 \\
\hline BAS Magnetic Storms & bas_magnetic_storms & index & 1992-Van-08 & 2002-Dec-28 & active & 372 \\
\hline NOAA SRS Active Regions & srs_list & index & 1996-Jan-02 & 2005-May-16 & active & 31929 \\
\hline SoHO Campaign & soho_camp & list & 1996-Mar-06 & 2009 Jan-01 & active & 1099 \\
\hline NOAA Daily Solar Data & dsd_list & index & 1994-Jan-01 & 2005-May-16 & active & 4154 \\
\hline SIDC Smoothed Monthly Sunspot No. & sidc_sunspot_number & index & 1749-Jul-01 & 2004-Oct-31 & active & 3064 \\
\hline DRAO $10.7 \mathrm{~cm}$ Radio Flux Monitor & drao_10cm_flux & index & 1996-Feb-14 & 2005-May-15 & active & 10138 \\
\hline
\end{tabular}

Figure 2. List of available catalogues.

\section{Added Values}

\subsection{Solar Event Catalogue}

The aim of the Solar Event Catalogue (SEC) is to propose a unique interface to get informations from several event catalogues scattered in various places. Link to SEC can be found at http://www.egso.org/software.

Figure 2 shows the events catalogues accessed by EGSO (at any time the status of access is indicated). The time extension of the informations contained in those catalogues is available on the web site. 

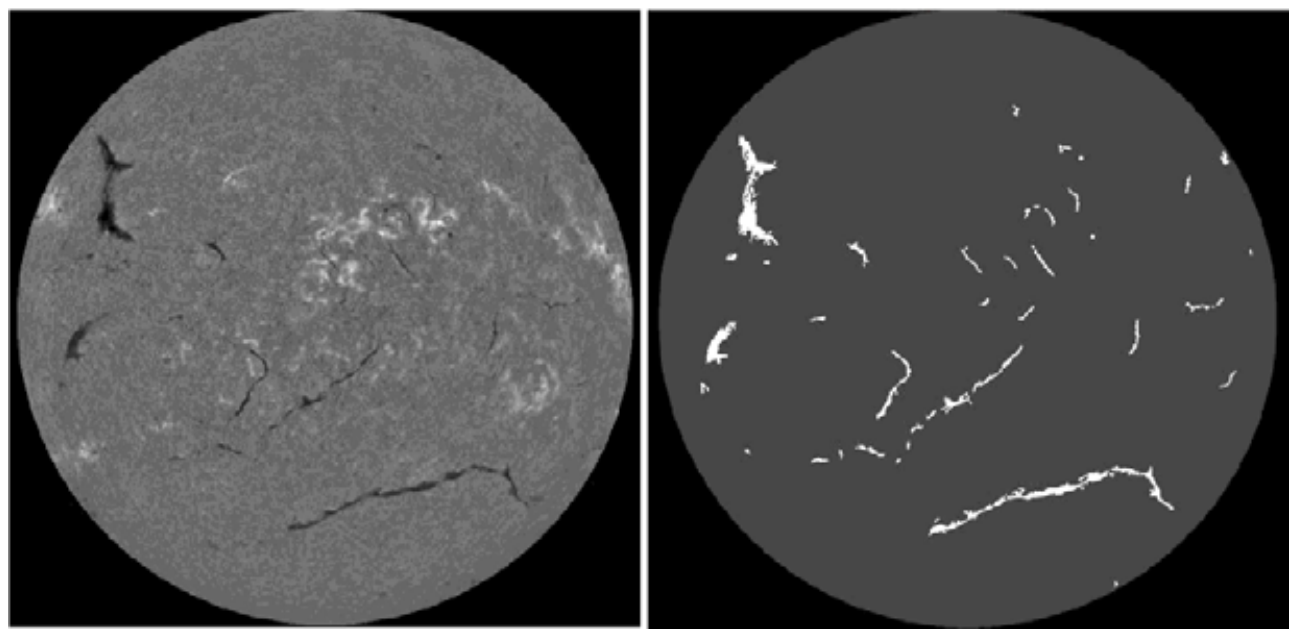

Figure 3. Example of filaments' automatic detection. Left image: original image. Right image: filaments detected.

\subsection{Solar Feature Catalogue}

One workpackage of EGSO was totally devoted to automatic feature recognition. This recognition occurs in two steps. First, a cleaning process of the image is made, then the feature recognition code is applied.

The cleaning process consists in a removal of several defects common in ground-based observations, such as non uniform transparency of the sky, straight lines due to dust on the entrance slit of the instrument... (Zharkova et al. 2003a).

Most of the recognition codes have been developped using Meudon spectroheliograph daily observations. The codes have been developed for filaments (Fuller et al., 2005), prominences, sunspots (Zharkov et al., 2003) and active regions (Zharkova et al., 2003b) (see figure 3 for an example of filament detection). The result of the detections is stored in a database, the Solar Feature Catalogue (SFC, see http://www.egso.org/software for a link towards the SFC) (Zharkova et al., 2005). It contains various informations on the cleaning and detection processes, but mainly a complete and precise description of each structure, as well as a representation of the structure either as a chain code, or as a raster scan.

With those informations, it is then possible to superimpose features on any image of the Sun, eventually after applying a shifting factor due to the difference in observing times as one can see on figure 4 .

\section{Queries}

\subsection{Classic query}

As in a usual database, one can make, via EGSO, a standard query, asking for observations available in a given time interval. The difference is that several archives are accessed simultaneously (see the list on figure 5), and the user can then choose which instruments are needed, then retrieve interesting observations. 

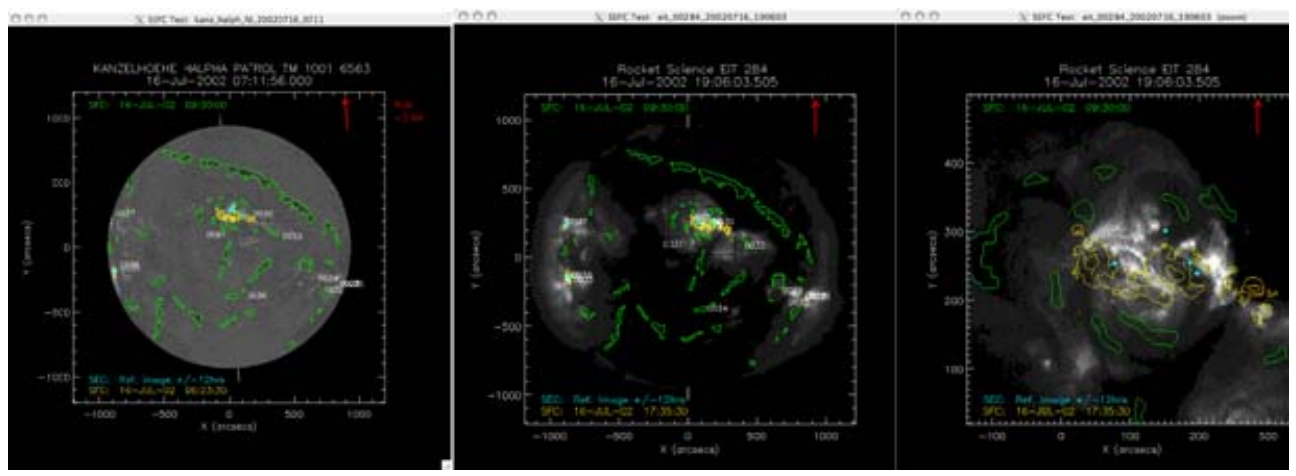

Figure 4. Events informations and structures automatically detected superimposed: Left: on a Kanzelhoehe observation in $\mathrm{H} \alpha$. Filaments are plotted in green, sunspots in yellow, coming from the Solar Feature Catalogue; blue and white are respectively flares and active regions obtained from the Solar Event Catalogue. Center: superposition on a UV full Sun image. Right: Close-up view of the very active region.

\begin{tabular}{|c|c|c|}
\hline Observatories / Instruments & Location of Archives & Access type \\
\hline Space instruments & 2 & \\
\hline CDS, EIT, LASCO, MDI, SUMER, SWAN, UVCS & $\begin{array}{l}\text { Catalogue : IAS, France } \\
\text { Data : SDAC, USA }\end{array}$ & $\begin{array}{l}\text { SQL } \\
\text { VSO-WS }\end{array}$ \\
\hline $\begin{array}{lr}\text { SOHO / } \\
\text { CELIAS, COSTEP, ERNE, GOLF, VIRGOS }\end{array}$ & RAL, GB & SQL \\
\hline SMEI / Coriolis & NSO, USA & HTTP \\
\hline Yohkoh & SDAC, USA & FTP \\
\hline RHESSI & HEDC, Suisse & FTP \\
\hline GOES-12 / SXI & NGDC, USA & HTTP \\
\hline \multicolumn{3}{|l|}{ Ground-based instruments } \\
\hline $\begin{array}{r}\text { Meudon, Nancay, Pic du Midi } \\
\text { THEMIS }\end{array}$ & $\begin{array}{l}\text { BASS2000/Meudon, France } \\
\text { BASS2000/Tarbes, In progress }\end{array}$ & SQL \\
\hline Nobeyama & Japan - In progress & FTP \\
\hline MLSO & HAO, USA - In progress & vso Ws \\
\hline BBSO, KANZ, YNAO, GONG, HSOS, OACT & Big Bear, USA & FTP \\
\hline EVANS, KPVT, MCMATH & NSO, USA & vso Ws \\
\hline
\end{tabular}

Figure 5. List of archives accessed with EGSO. The access type is also indicated. Note that some archives are reached through the US Virtual Solar Observatory.

\subsection{A new way to look at observations: the Event Query}

What really offers strong new possibilities is the event query, shown on figure 6 .

The first step consists in selecting a time interval (in order not to get too many results!) and a kind of event (e.g. M flares, proton events, ...). EGSO sends back a list of events corresponding to the query during the time interval (together with, if asked for, a GOES $\mathrm{X}$-ray plot for the considered period of time).

From that list, it is possible, by clicking on an orange button near the event, to open web pages containing informations of the event. Or tick off the square near the event in order to select it. EGSO then returns a list of instruments that have been observing during at least one part of the duration of the event. 


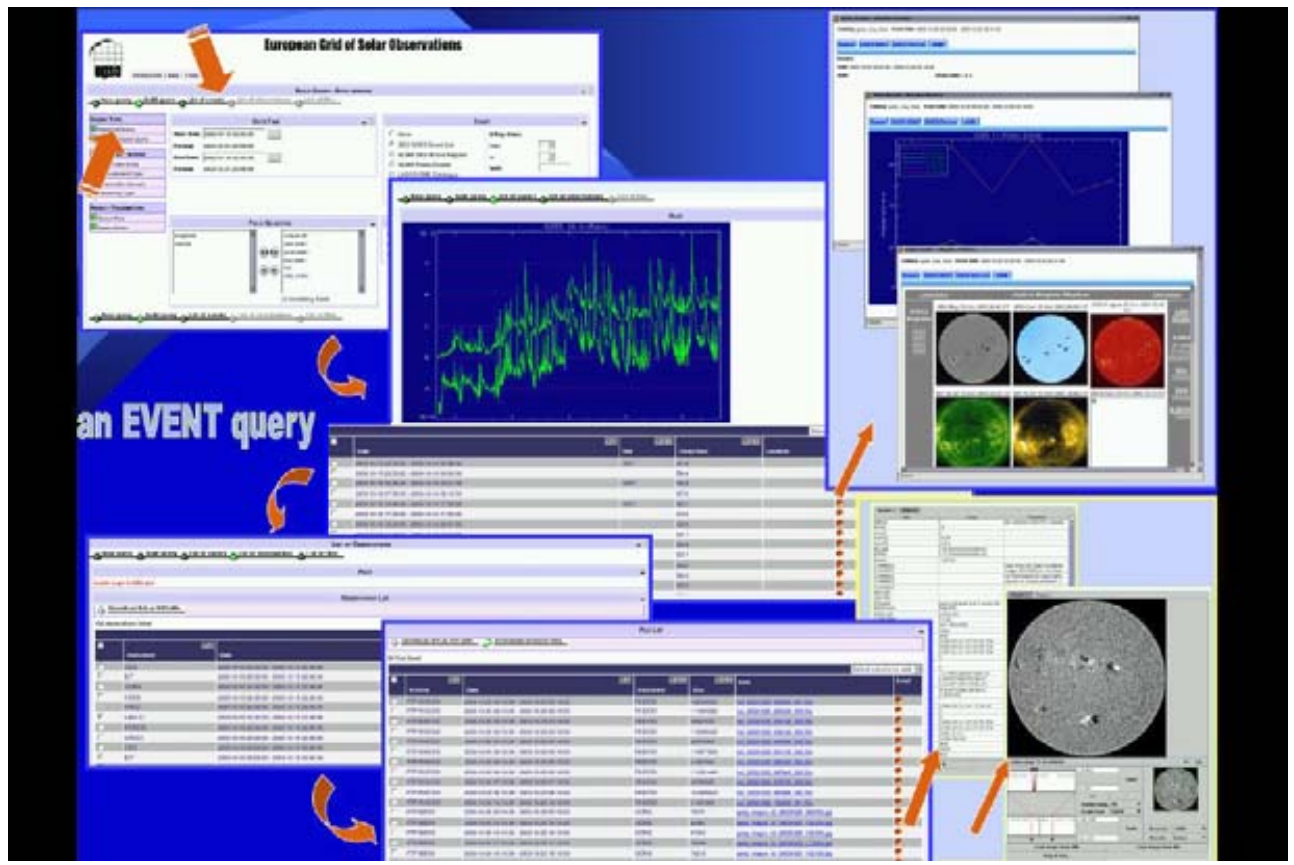

Figure 6. Detail of an event query (see text, 5.2, for explanation).

Then one has to select instruments needed and EGSO sends back a list of files. By clicking on the orange button near the filename, a Java application is launched that allows visualization and basic processing of the image (such as change contrast), and reading of the header for a FITS file.

Then, by selecting files user can download them locally.

\section{Conclusion}

EGSO provides a new way to access observations, and a new way to build queries, taking advantage of the mix of Solar Event Catalogue and Solar data. Moreover, an inclusion of the Solar Feature Catalogue in the future within EGSO, will allow once again new ways to query data. The global behaviour of the Sun can then be grasped. But for taking full advantage of all those opportunities, it's important to have a full longitude coverage for Sun's observation which is lacking nowadays.

\section{Acknowledgements}

EGSO has been supported by the European Community under grant number IST2001-32409.

\section{References}

Fuller N., Aboudarham, J., Bentley, R.D. 2005, Solar Phys. 227, 61

Hill, F., Csillaghy, A., Bentley, R.D., Aboudarham, J., Antonucci, E., Finkelstein, A., Ciminiera, L., Gurman, J.B., Scholl, I., Pike, D., Zharkova, V.V. 2002, in "Virtual Observatories", edited by Alexander S. Szalay, EGSO in need for a global schema, Proceedings of the SPIE (22-28 August 2002, Hawaii, USA), Volume 4846

S. I. Zharkov, V. V. Zharkova, S. S. Ipson, A. Benkhalil 2003 An Automated Recognition of Sunspots from the Ca K1 and White Light Solar Images and Corresponding Magnetic 
Structures from the SOHO/MDI Magnetograms, Proceedings of the AISB'03 Symposium on Biologically-inspired Machine Vision, Theory and Application, University of Wales, Aberystwyth, 7th - 11th April 2003, pp. 74-84, Proceeding ISBN 1-902956-33-1, 2003

V.V. Zharkova, S.S. Ipson, S.I. Zharkov, A. Benkhalil, J. Aboudarham, R.D. Bentley 2003, Solar Phys. 214, 89

V. Zharkova, A.Benkhalil, S. Zharkov, and S. Ipson 2003 An Automated Detection of the 3DStructure of Active Regions from Full Disk Solar Images, Proceeding of the Computational Engineering in Systems Applications, CESA'2003, Lille, France, July 9-11 2003, Proceeding ISBN 2-9512309-5-8

Zharkova V.V., Aboudarham J., Zharkov S., Ipson S.S., Benkhalil A.K., Fuller N. 2005, Solar Phys. 228, 361 